\title{
The Establishment and Thinking of Chinese Medicine Standard Decoction
}

\author{
$\mathrm{Xu} \mathrm{ZHANG}^{1, \mathrm{a}}$ and Ming-san MIAO ${ }^{1, *}$ \\ Henan University of Chinese Medicine, Zheng zhou, China. \\ azhangxuyaoli123@163.com \\ ${ }^{*}$ Corresponding author
}

\begin{abstract}
Keywords: Chinese medicine standard decoction, Influencing factors Industry standard
\end{abstract}

\begin{abstract}
Through the research on the development history of Chinese medicine traditional decoction, the Chinese medicine decoction was compared with the scrambled and fried granules, and the effects of Chinese medicine decoction were discussed from the perspectives of quality and quality of decoction, boiling utensils and fire temperature. Quality of the proposed, and the concept of Chinese medicine standard decoction, to ensure the efficacy of Chinese medicine decoction processing methods for the establishment of traditional Chinese medicine standard decoction industry standard to provide a theoretical basis.

Chinese medicine decoction, also known as decoction, refers to the Chinese medicine under the guidance of the theoretical system, will be thin or broken single herb, or in accordance with the physician prescription compatibility of a variety of Chinese medicine Pieces, adding water, into a specific decoction after boiling for some time, discarding the residue, taking concentrated juice to made of liquid medicine. Decoction mainly use water as a solvent, but sometimes may use wine, vinegar, honey and other fry related to the drug and the need for treatment to promote the active ingredients in the drug dissolution.It is currently widely used in clinical practice of a dosage form. Although the use of decoction in the clinical use of a wide range, but there are clinical application of personalized decoction caused by the traditional uniform quality of the decoction poor, ineffective, mass production and there is no uniform standard, the quality of production can not be organic unity and other issues The Therefore, based on the traditional Chinese medicine traditional decoction preparation and characteristics, this paper proposed the concept of traditional Chinese medicine standard decoction, that is aimed at curing the product form, standardized production process, for the establishment of traditional Chinese medicine standard decoction production and application of industry standards to provide a theoretical basis.
\end{abstract}

\section{The History of Chinese Medicine Decoction}

Chinese medicine is produced and served in the production and living practice of the ancient Chinese working people. "In the era of iron-free, before cooking decoction, people chewed the medicinal herbs into small pieces, in order to facilitate the decoction "drug with the out" [1]. To the Han Dynasty," Treatise on Miscellaneous Diseases" written by Zhang Zhongjinga recorded a lot of classic prescription. The method of some prescriptions is crushing the pieces, and making into decoction."Qianjin Yi Fang" written by Sun Simiao recorded the phrase "powder with water" firstly, and this preparation in the Song Dynasty had been widely used. Cooking with a decoction and powder characteristics, can reduce the amount of drugs 
to ensure efficacy. However, because the powder with water and water decoction is different, which is the drug after crushing together with the crushed together into the water decoction, so the liquid is turbid, and the taste is very strong. For children and older patients, it is not easy to accept.

With the improvement of living standards and the pace of life, patients have a higher demand with Chinese medicine preparation efficacy, taste, quality and other aspects. So Chinese medicine formula particles came into being. Chinese medicine formula granule is made by single flavor of Chinese herbal medicine by the extraction and concentration, under the guidance of the theoretical system of Chinese medicine for the clinical use of Chinese medicine particles, with uniform specifications, unified dose, unified quality standard Chinese medicine. Its biggest advantage lies in the quality control, water leveling, without boiling [2]. However, compared with the traditional Chinese medicine Pieces, the Chinese medicine-free fried particles ,fundamentally speaking, do not have the traditional Chinese medicine "multi-component multi-target "Characteristics because of the processing process by adding a variety of accessories and chemical composition than the single tablet. So compared with the traditional Chinese medicine decoction, there are still some differences in its clinical efficacy.

\section{Factors Affecting the Quality of Chinese Medicine Decoction}

The quality of Chinese medicine decoction is affected by many factors. Generally speaking, it is affected by three factors: Prepared drug in pieces factor, Boiling condition factor and Patient factor.

\section{Prepared Drug in Pieces Factor}

Prepared drug in pieces factor is the most direct impact on the efficacy of traditional Chinese medicine decoction factor. There is the" genuine regional drug" in traditional Chinese medicine culture, and many old Chinese medicine also pay attention to the origin of prepared drug in pieces, quality and so on. Adulterated prepared drug in pieces or counterfeit goods, in principle, prohibit the drug, otherwise it is invalid lightly, while it would injured life. Different areas of the production of prepared drug in pieces may have different efficacy, such as pregnant with achyren flat taste bitter, can fill liver and kidney, strong bones and bones, Achyranthes bidentata can Tongli joints, Qufeng diuretic; cinnabar Qingxin town of God, The toxicity is large, medicine must be very cautious. Powder anti-self [3] has Qufeng analgesic effect, wide anti-containing aristolochic acid, with renal toxicity. Prepared drug in pieces of the concoction will also change the drug bias, affecting the efficacy of decoction, such as ephedra. The raw ephedra sweat solution strong, honey sunburn epiphytic cough and asthma, linen force slow, suitable for children and the elderly and other frail patient. Therefore, the quality of prepared drug in pieces is good, and the effect will be significant. We must do a good job in the production, processing, transportation of the Chinese medicine industry chain in all aspects, in order to ensure the quality of prepared drug in pieces.

\section{Boiling Condition Factor}

Decoction Equipment. Traditional Chinese medicine decocting equipment are the casserole or pottery for the high temperature, and enamel appliances can also be used, while people cannot use iron, aluminum and other appliances, because these containers are easy to produce chemical reaction with the liquid, which could affect the quality and effectiveness of decoction, even harm the human body ${ }^{[4]}$. At present, 
large quantities of Chinese medicine decoction are used in the decoction machine are equipped with stainless steel liner. On the impact of different materials on the dissolution rate of effective substances, Some scholars have compared the electronic Zisha pot, electronic ceramic pots and Induction Cooker stainless steel utensils on the effect of silver Alice scattered decoction[5], and measured in different decoction boiled 20min after the paste rate and chlorogenic acid content. The results showed, Electronic pottery pot, electronic pottery pot and induction cooker stainless steel utensils after decoction and chlorogenic acid content were significantly different; electronic purple pot and electronic pottery pots of the rate of no significant The results showed that there was a significant difference in the content of chlorogenic acid after boiling, and the results were excellent for the selection of electronic ceramic pots.

The Source of Fire. Ancient life conditions are limited. Decoction of fuel often uses firewood. There are many types of wood. Traditional decoction often use coal furnace. Boiling liquid temperature is generally about 100 degrees Celsius. Liquid temperature increase slowly, which is conducive to the dissolution of active ingredients of drugs. Modern patients mainly use natural gas and induction cooker, while Chinese medicine on behalf of the frying institutions commonly used high temperature and high pressure decoction equipment. This modern boiling equipment heating fast, especially the decoction machine. The container temperature could be up to $120{ }^{\circ} \mathrm{C}$, and working pressure could reach $203 \mathrm{kpa}$, and the effective material dissolution rate is much higher than the traditional boiling method of liquid [6]. But whether there will be the same dose of drugs under the conditions of taking too much effective substances and produce excessive drug use situation, also to be discussed.

Production process. The Production process is the most directly link to the quality of decoction, and the duration of soaking, the duration of decoction, the order of the prepared drug in pieces, the number of decoctions and so on need to be determined according to the type of decoction contained in the prescription. Chinese medicine soaking duration is generally $30 \mathrm{~min}$,.For plants, which take the flowers, leaves, stems and other herbs into medicine, the general duration should be 20-30min. For plants, which take root, rhizome, fruit, seed into medicine, the general duration should be $60 \mathrm{~min}$, in order to facilitate the dissolution of effective substances. Some scholars have shown that different immersion time has a significant effect on the shellfish dissolution rate of Fritillaria thunbergii, and the Fischer-Tropsch Fritillaria Fischer-Tropsch Fibroin is the most effective. [7]

In terms of boiling duration, it is not good as long as possible. According to the efficacy of classification, tonic medicine should be long boiled, and could be added first. For sweating solution table medicine, because the active ingredients often volatile oil, it should not be long boiled, the best be added later. According to the different drug parts, stone crushed long after the fry, you can increase the effective material dissolution rate, the smell of aromatic leaves of the whole plant herbs can be fried in the first 5-10min into the decoction. In addition to some special medicinal materials need to be fried or chilled to take, such as some valuable herbs, in order to avoid the dissolution of the active ingredients absorbed by other herbs, more than a single fried, some sugar content of high quality sticky herbs, Taking. Some scholars have statistics on the treatment of lung cancer, the statistical results show that the role of cough and phlegm played bitter almond, in the decoction 16-20min, the main active ingredient amygdalin dissolution rate reached the threshold; from the role of blood circulation the optimum boiling time of Radix Rehmannia glutinosa was $20 \mathrm{~min}$. The content of active ingredient in Rehmannia glutinosa was inverted with time, and the 
highest content was at $1 \mathrm{~h}[8]$.

In general, Chinese medicine to be boiling 2-3 times, is the effective ingredients fully dissolved, the decoction combined to take. Some scholars have shown that the traditional casserole in accordance with the traditional method of decoction Liu Wei Di Huang Tang, and the use of modern decoction machine for boiling, the liquid, the effective substances to paeonol, casserole decoction of the liquid The content of paeonol was higher than that of the decoction machine, and there was significant difference, and the time of immersion of medicine was prolonged. The liquid of the decoction group was not significantly better than that of casserole group ${ }^{[9]}$.

\section{Patient Factors}

The patient is the main body of Chinese medicine decoction, so the dose of medication, the frequency of medication, and the effective time of the drug and the decoction after taking the effect will have an impact. Clinical moderation can be a daily oral 2-3 times, and the condition of the patients, according to the needs of the disease, need take several times to ensure the continued use of drugs in the body in daily, to speed up the body recovered, which is conducive to early rehabilitation. In terms of efficacy classification, tranquillizing formula is suitable for administered before bed time, and supplementing and boosting formula should be administered at empty stomach, in which is conducive to digestion and absorption. Digestive formula and stimulating drugs on the stomach should be taken after meals to reduce the gastrointestinal tract of the stimulus. Drastic diuretics formula and anti-helminthes formula should be taken in fasting [10].

The pace of modern life to speed up, patients often choose a one-time or a longer course of drugs decocted into decoction, cold, in order to make the liquid content of the effective substances and ingredients stable, when used to disable the hot water, Hot orally. At present, there is no relationship on the content of drug substance and the storage time of traditional Chinese medicine decoction. The method of evaluating the shelf life of traditional Chinese medicine decoction is also carried out for microbiological examination. The results showed that the number of bacteria was relatively low at $2-8{ }^{\circ} \mathrm{C}$, and the results showed that the number of bacteria was relatively low. So low temperature is more conducive to the preservation of liquid [11].

\section{Chinese Medicine Standard Decoction Design}

In the preparation of Chinese medicine herbal medicine, the preparation of Chinese medicine decoction has strict requirements, decocting conditions on the clinical efficacy of decoction has a crucial impact. Modern research also shows that decoction of Chinese medicine decoction of the effective substance dissolution rate A great impact, so the decoction of Chinese medicine decoction is to ensure that the decoction of Chinese medicine decoction of the key factors. As mentioned above, in the production process, the control of the effect of Chinese medicine decoction and the dissolution rate of active ingredients are mainly decocting equipment, soaking time, boiling time and other factors. Therefore, in order to regulate the high-volume production of Chinese medicine decoction, the establishment of Chinese medicine decoction standard SOP is practical and is much needed. SOP quality control standards should be established a complete set of quality control standards, modern research and production from the effective material dissolution rate and content to control the production conditions. However, traditional Chinese medicine is different 
from the chemical drugs, is a multi-component work together, single or several components are not necessarily a positive correlation with the efficacy of decoction. The most convincing evaluation criteria is clinical efficacy, so only the determination of active ingredients as a quality assessment method is not perfect, but also should be combined with pharmacodynamic research to determine the final help in the establishment of Chinese medicine decoction SOP.

\section{Problems and Prospects}

Chinese medicine comes from the production and living practice, and serves the production and life practice. Since the Shennong tasting Baicao, Yi Yin a decoction so far, Chinese medicine for the health of the Chinese people escort for thousands of years, ancient physicians Chengtu Tu new, in the continuous medical practice enrich and develop the theoretical system of Chinese medicine, to modern, In different and ancient social background, the theory of Chinese medicine and practical application has a leap of innovation and development. However, the dialectics of materialism that we have to dialectically look at the problem, Chinese medicine not only has its own advantages, but also has its own shortcomings hinder its further development. (2) The taste is poor, for children or elderly patients Patients, the Chinese medicine decoction tastes bit, especially the scouring, which will lead to poor compliance of patients, affecting the clinical efficacy of decoction; (3) cumbersome cooking steps, traditional Chinese medicine decoction process is more stress, time-consuming and laborious (4) At present, there is no uniform production standard for the production of large quantities of decoction, and the quality controllability is poor, and the boiling process can not be repeated. (4) At present, there is no uniform production standard for the production of large quantities of decoction.

Based on the above status quo, the author puts forward the concept of Chinese medicine standard decoction, that is, according to prescription Chinese medicine Pieces different medicine parts or different effects, follow the Chinese medicine in the first fried after the next decoction principle, the development can be applied to the mass production of repeatable Of the boiling conditions, in order to achieve quality control, quality and stability, significant effect, the output and quality, efficacy of organic unity. With the existing standard of Chinese medicine particles ${ }^{[12]}$, the Chinese medicine standard decoction is Chinese medicine Pieces as raw materials, will be the characteristics of Chinese medicine and modern products combined with the product. With the Chinese medicine standard decoction production industry standards to establish and promote the Chinese medicine decoction of this traditional and widely used in the research and development of Chinese medicine formulations will be full of vitality.

\section{Acknowledgement}

These research is based on National international cooperation base (Guokewaihan2016-65), Zhongyuan scholar (162101510003), Henan Province production and research projects (142107000039). 


\section{References}

[1]Li Rui, Zhai Huaqiang, Tian Weilan, Hou Jiru, Jin Shiyuan, Wang Yongyan.The Historical Origin of Traditional Chinese Medicine and Its Contrastive Analysis with Modern Formula Granules[J].Journal of Chinese Medicine,2016,(05):965-969.

[2]Meng Fan Chen, Guo Zhaoan.Comparison of traditional Chinese medicine granules with traditional Chinese medicine decoction.Chinese Journal of Medical Science.2015,(03):89-90+105.

[3]Jia Caifeng, Bu Yunge, Deng Shuiqing, Zhang Qingli, Zhang Guowei.Investigation on the Third Party Testing Requirement of Chinese Herbal Medicine Pieces in Hebei Traditional Chinese Medicine Hospital[J]. Medical Research and Education. 2017(02)

[4]Cheng Jingyan.Treatment and Efficacy of Traditional Chinese Medicine Decoction [J]. Chinese medicine. 2012(14)

[5]Wu Jinhua, Zheng Guoshen, Wu Yi, Li Jincai, Chen Yuanda.Effects of Different Organs on the Effect of Yinqiao Powder[J]. Chinese pharmacy, 2011, (11):997-998.

[6]Gu Yuanmei.Effects of Different Decoction Methods on the Dissolution of Compound Danshen Decoction [J].Chinese National Folk Medicine, 2017, (03):43-44.

[7]Jin Yuqin, Zeng Guowu, Wang Longhu, Wang Zengshou.Effects of Different Soaking Time on Extraction of Active Ingredients of Fritillaria thunbergii[J].Chinese medicine,2012,(20):24-25.

[8]Zhang Zeyuan, Sun Jianfeng, Zhong Dan, Zhang Yifan, Xia Zhiyu, He Chengshi.Effect of Decocting Time on the Quality of Decoction of Lung Cancer[J].Laozhen Guoyao medicine,2014,(12):2916-2917.

[9]Qi Huizhen, Huo Bingjie, Zhou Xiajin.Comparative Study on Decoction Rate of Liuwei Dihuang Decoction by Two Kinds of Decoction [J]. Hebei Medicine, 2016, (08): 1262-1263.

[10] Zhou Chufu. Talking about the effect of traditional Chinese medicine decoction factors [J]. Chinese Journal of Modern Medicine, 2013, (05): 123-124.

[11] Liang Xuezheng, Chen Huigong, Tang Yongchen, Tao Hong, Huo Wei. Microbiological limits of bagged Chinese medicine decoction[J]. Journal of Guangxi University of Traditional Chinese Medicine, 2013, (03): 48-50.

[12] Zhang Shuihan, Liang Xuejuan, Liu Hao, Wan Dan, Toda Guangyin, Chen Lin, Zhou Rongrong, Huang Luqi. Traditional Chinese medicine standard decoction scientific issues [J]. Chinese Journal of Traditional Chinese Medicine: 1-15. 